\title{
KNOWLEDGE AND ATTITUDE OF REHAB SCIENCES STUDENTS TOWARDS BYSTANDER CARDIOPULMONARY RESUSCITATION
}

\begin{abstract}
\section{BACKGROUND AND AIMS}

Sudden cardiac arrest is the major health related issues with high risk of death and long-term disabilities if not treated promptly through immediate cardiopulmonary resuscitation (CPR). Therefore, this study aims to explore the knowledge and attitude of rehab sciences students towards bystander CPR since it is one of the life-sparing intervention in response to sudden heart failure that consists of chest compressions followed by defibrillation. METHODOLOGY

A cross-sectional survey conducted on 136 rehab sciences students recruited via convenience sampling technique. A self-administered questionnaire consists of understanding towards bystander cardiopulmonary resuscitation was distributed via email or Google forms to record participants' responses.
\end{abstract}

\section{RESULTS}

A total number of 136 responses received with mean age of $22.34 \pm 3.92,109$ (79.2\%) females and 27 (19.8\%) males. It was revealed that $83.1 \%$ students have understanding regarding CPR whereas only $62.7 \%$ approached the certified course While the $38.2 \%$ acquired knowledge by medical personnel provided by school/university.

\section{CONCLUSION}

It was concluded that majority of students have understanding and had a positive attitude to learn and perform the technique. However, the knowledge was found to be scared and the students are not confident in performing CPR. Hands on skill must be promoted for better care.

\section{KEYWORDS}

Cardiopulmonary, Resuscitation, Heart, CPR, Health, Physiotherapy, Defibrillator
Sana Mohammad Yousuf

Physiotherapist

Freelancer

sananasir611@gmail.com
[Yousuf SM, Knowledge And Attitude of Rehab Sciences Students Towards Bystander Cardiopulmonary Resuscitation.Pak.j.rehabil.2021;1 0(1)34-41]

10.36283/pjr.zu.10.1/006 


\section{INTRODUCTION}

Cardiopulmonary resuscitation (CPR) is an effective lifesaving emergency procedure that consists of cardiac compressions, rescue breathing and cardiac defibrillations in response to sudden heart and respiratory failure. Worldwide, the prevalence of heart failure is 64.34 million cases (8.52 per 1,000$)$. However, in US, 5.7 million people have suffered with cardiac arrest, but the future projections are very crucial since it is expected that by 2030 more than 8 million people will have this ${ }^{1}$. Prompt CPR and defibrillation within 3-5 minutes after arrest can increase the survival rate by $50 \%$ and also prevent the survivor from fatal lifelong disabilities ${ }^{2,3}$. Therefore the strategic planning should be developing to appraise the Basic life support (BLS) and CPR skills in undergraduate medical students ${ }^{3}$.

In various emergency conditions, CPR is a significant life-sparing intervention to protect the individuals from fatal consequences. It consists of a proper sequence of activities needed for effective resuscitation including chest compression and rescue breath that reestablish heart contraction, thus enable proper oxygen conveyance and maintaining cerebral circulation ${ }^{4}$. The effectiveness of CPR is based on prompt identification of heart failure and initiation of the chest compressions immediately. Evidences claimed that rapid and prompt attempt of CPR within 10minutes after postponed circulation increase patient's rate of survival and assure the cerebral perfusion thus preventing from long term disabilities and stroke ${ }^{5}$.

The protocol of CPR consists of combination of rescue breaths and chest compressions which are planned to restore heart contractility and blood flow in a person who has suffered with cardiovascular or respiratory event ${ }^{6}$.
Cardiovascular/respiratory event is a typical crisis in the adults as well as in the neonates ${ }^{2}$. However, the multiple studies provided the evidences that the initial 4-8 minutes after the heart arrest is the most pivotal period where resuscitation intercession is most required. On the other hand, the appropriate sequence and correctly applied technique by a trained bystander is equally important in an emergency situation of cardiac arrest. Moreover, a prompt attempt of CPR can keep the person alive until a clinical expert in a hospital emergency take control over the consideration and further intervene through advanced cardiac life support. Moreover, in various studies it was found that insufficient knowledge about CPR leads to fatal consequences that may indulge the applicants into different medico-legal complications. Therefore, the proper training regarding BLS is much needed in our country specifically by healthcare providers so as to prevent the person from lifelong disabilities and additionally from death ${ }^{7}$. Besides this, performing a potent CPR is based on updated knowledge, experience and potential of an applicant8. Worldwide various medical and non-medical universities incorporated BLS and CPR as a significant part of their clinical educational plan that amplify the potential of their students and make them confident in applying cardiopulmonary resuscitation ${ }^{9}$. While the competency in providing CPR is viewed as a central expertise among different healthcare providers including doctors, nurses, physical therapist and occupational therapist. Along with these, further Studies have done to evaluate the nature of CPR performed by different medical professionals. However, it was not realized that whether the level of information and practices of CPR is compromised due to inadequate training or there were any other factors responsible for incompetent and poor quality $\mathrm{CPR}^{5}$. Whereas, the quality and 
extent of training in bystander CPR fluctuates in various nations and the major cause of this variation in CPR training is probably the difference in training system and instruction given ${ }^{10,11}$ However, the ability of the practitioners to understand and perform CPR among various nations is likewise significant. Many factors has been identified in which no training opportunity to learn CPR is the most highlighted one while the fear to apply technique incorrectly, and many other reasons are pronounced that confined the person to learn and apply CPR ${ }^{12,13,14}$. In contrast, number of studies suggested that willingness, knowledge and self-adequacy of students increase after CPR training ${ }^{15,16}$. In another report published by China's Tianjin colleges demonstrated the reasons of not able to perform CPR in which they highlighted the lack of confidence, fear to be indulge in law related issues, scared to have disease transmission and feeling uncomfortable are the main causes ${ }^{17}$. However, there is a significant scarcity of knowledge and awareness related to CPR among students of different disciplines ${ }^{17,18}$. In addition to these factors another study emphasized the role of awareness and training programs in promoting and escalating the good CPR practices as well as enables the students to apply effective bystander $\mathrm{CPR}^{19,20}$. Furthermore, another investigation revealed incredibly poor information regarding BLS among doctors and nurses who are considered to be the main applicants accounted for $\mathrm{CPR}^{21}$. A survey conducted in Pakistan revealed that majority of clinical professionals has inadequate knowledge of BLS therefore promoting BLS training may cultivate knowledge and thus enabling the healthcare provider in intervening successful resuscitation ${ }^{3}$.

Therefore, in the light of these factors our aim of study is to explore the knowledge and attitude of rehab sciences students towards bystander cardiopulmonary resuscitation and to strategize the best CPR training program for our students that ensure effective and safe delivery of this life saving technique in future.

\section{METHODOLOGY}

Study Setting: Data was collected from primary and tertiary care institutions of Karachi including Institute of Physical Medicine and Rehabilitation (IPM\&R), Liaquat National School of Physiotherapy (LNSOP) and Ziauddin College of Rehabilitation Sciences, North and Clifton campuses respectively.

Target Population: Undergraduate rehab sciences students.

Study Design: Cross-sectional study.

Duration of Study: 6-8 months.

Sampling Technique: Non-Probability Convenience Sampling Technique. Sample size was calculated by using Open EPI software version 3.01. Therefore, at anticipated frequency of $50 \%$, Confidence Interval of $95 \%$ with margin of error at $5 \%$, a sample size of $n=136$ was calculated

\section{Sample Selection}

\section{Inclusion criteria}

- Both genders

- 3rd, 4th and 5th year students of physical therapy and occupational therapy

\section{Exclusion criteria}

- Students who refused to participate,

- 1st and 2nd year students of physical therapy and occupational therapy

Data Collection Tool: Data was collected through a self-administered questionnaire comprising of 23 questions based on demographics, knowledge and attitude towards bystander cardiopulmonary resuscitation. The questions mainly focus on the course of CPR, source of information, steps, training and some open-ended questions are there to evaluate the participant's response on varying options. 
Data Collection Procedure: Data was collected from different institutes of Karachi. At first, participants were recruited as per defined inclusion criteria. Prior to data collection, consent was taken from participants to ensure their voluntary participation. Further, a selfadministered questionnaire was made on Google Docs that constituted on participant's consent, demographic details and questions about knowledge and attitude towards bystander CPR that was distributed to students' online using email or through social media applications in order to assess their responses regarding the objective to analyze it further for results.

Data Analysis Strategy: Data was entered and analyzed using SPSS (Statistical Package for Social Sciences) version 20. The demographic characteristics of the participants were represented through frequency, mean and standard deviations whereas the participant responses were evaluated through frequency and percentage.

The researcher was provided complete information about the study to each and every participant before the start of the study. The information procured from the participants was kept as confidential information. Every participant has the full right of withdrawing the study at any time during the study.

\section{RESULTS}

A total number of 136 physical therapy and occupational therapy students participated in the survey with mean age of $22.34 \pm 3.92$ with 109 (79.2\%) females and $27(19.8 \%)$ males with highest responses involved from 19 to 26 years (87.5\%). The gender distribution chart is represented as Figure-1.

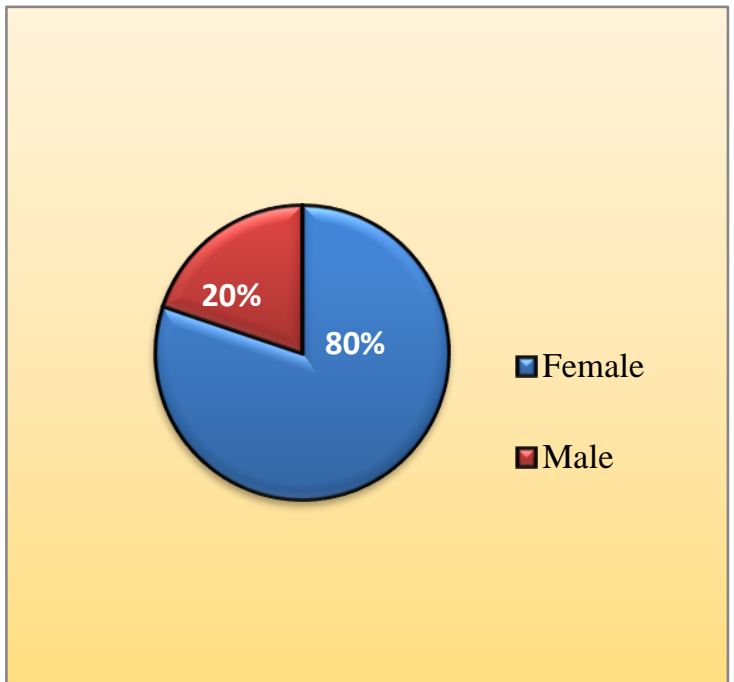

Figure-1 shows the percentages of male and female students

\section{CPR Knowledge and Training}

It was revealed that $83.1 \%$ students have understanding regarding CPR whereas only $62.7 \%$ approached the certified course While the $38.2 \%$ acquired knowledge by medical personnel provided by school/university as shown in Figure- 2 .

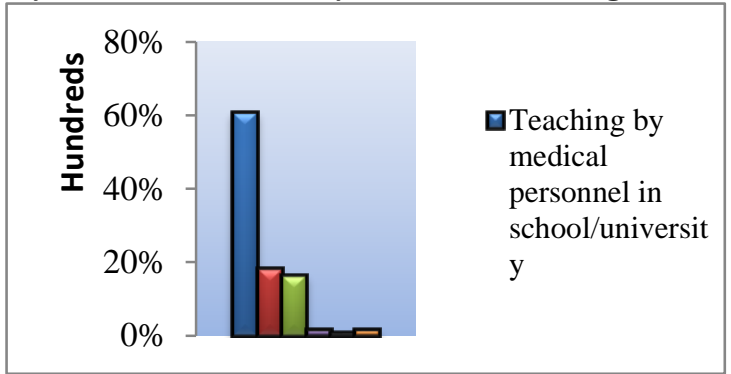

Figure-2 shows the source of attained information regarding CPR

However, (51.8\%) reported that the course was taken 1-3 years ago though (51.7\%) individuals know procedure and can perform CPR but (52.5\%) know the procedure but are non-trained. It was found that $56.7 \%$ of individuals would call for an ambulance when they encounter any situation that required CPR (Firgure-3) while $40 \%$ opt to begin CPR in this particular situation following correct sequence to maintain a patent airway (50\%), perform compressions $(35.7 \%)$ and artificial breathing (14.3\%). Unfortunately, only one respondent knew that if someone has cardiac arrest, an 
automated external defibrillator is used if available in case of cardiac arrest, begin CPR immediately and continue until a professional emergency medical service arrives.

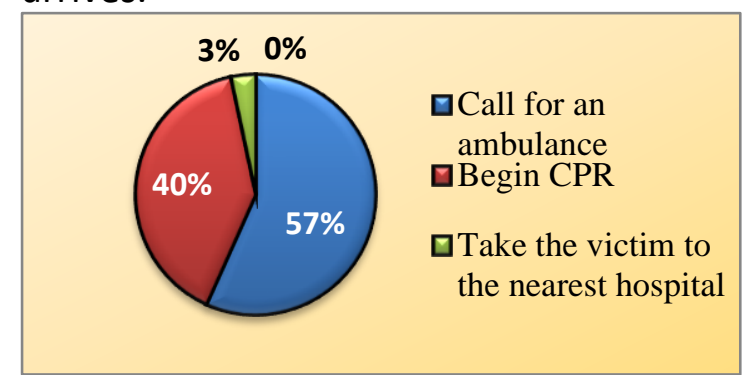

Figure-3 shows response of participants when encounter situation that requires CPR

Attitude-Willingness to Perform CPR A number of $(84.3 \%)$ students had a positive attitude to learn and perform CPR training as $(81.7 \%)$ have encountered a situation in their lives and (40.5\%) had resuscitate the person so (79\%) believed that a lifeless person without breath/heartbeat can be saved. On the other hand, (59.5\%) didn't opt for resuscitation but (65.5\%) will ask for help or proceed for CPR. On the other hand, majority of students (68.6\%) wants to pay for professional CPR training while almost all participants agreed for mandatory CPR training for students in undergraduate program.

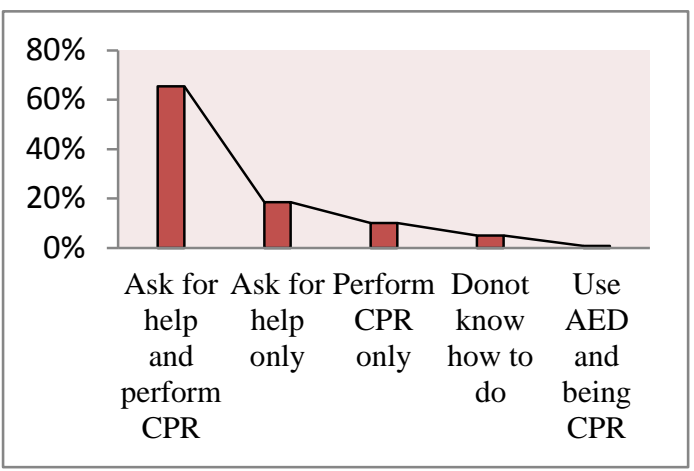

Figure-4 represents percentage of responses of participants if someone has cardiac arrest

Despite of the fact, number of participants was willing to spread awareness regarding CPR training (61.9\%) about currently available courses/training seminars $(13.6 \%)$ while $(23.7 \%)$ thought free training as the best method to increase the awareness of CPR (Figure-5).

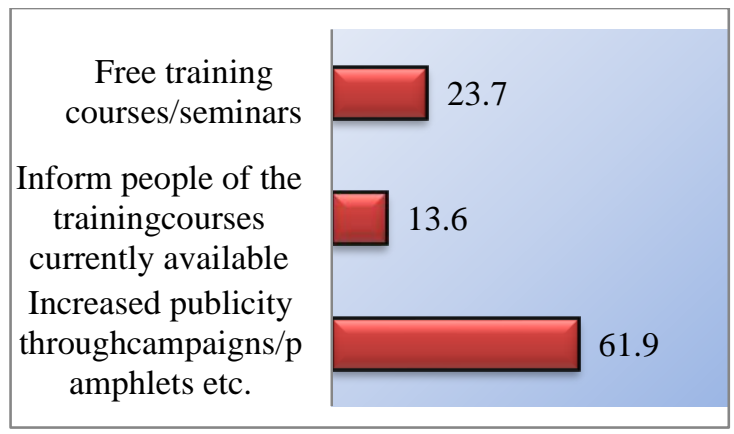

Figure-5 shows the participant's opinion regarding methods to increase the awareness of CPR

\section{DISCUSSION}

The findings of this study concluded that majority of students have understanding regarding CPR whereas (62.7\%) has taken the course due to mandatory work or graduation requirement and (84.3\%) students had a positive attitude to learn and perform CPR. As CPR is a potential life-saving protocol to protect individuals that might be suffering or experiencing heart failure therefore students must have adequate information related to it for better healthcare delivery in future. Similar to our study, another survey concluded that in China reported (90\%) students have understanding regarding CPR while $60 \%$ students attained information from medical personnel followed by accredited CPR training course (18.3\%)22. Moreover, CPR training rates in other countries were higher than those in reported in studies with university and secondary students 17,20

The component of attitude is essential while performing CPR. In our study it was revealed that majority of students believed that they have the ability to learn and perform CPR and showed willingness to take a training course that is similar to a study conducted in Saudi Arabia where most of the students were willing to perform CPR, whereas many of them were hesitated to perform 
Moreover similar to their findings, in our study most of the students are not familiar with the correct guidelines and majority of them are also unaware regarding $A E D{ }^{12,22,23}$.

In particular, studies have reported that $36.4 \%$ individuals previously trained for CPR knew the slandered processes while $48.3 \%$ knew the processes but could not perform CPR whereas only $35.7 \%$ knew the correct sequence ${ }^{1,21,24,25}$. Despite of the fact, in case of sudden cardiac arrest, CPR may make a significant difference. Therefore, to cater incidents of cardiac arrests outside of the hospital, comprehensive population education irrespective of qualification, experience and expertise will contribute significantly.

Moreover, it was further revealed in our study that most of the students confirmed about that if they witnessed a family member/stranger confronting cardiac arrest; in that case they will perform CPR to both of them. However, few of them applied CPR on a family member and $(2.5 \%)$ on strangers only, whereas the lack of BLS skills was the most frequent reason given for not initiating CPR in a given situation. Consequently, to manage the identified issues short refresher courses in addition to basic course regarding CPR should be announced to assure their practices according to the updated guidelines. Furthermore, the local peoples should be trained regarding the first aid. The barriers to CPR performance reported by the respondents included fear of legal issues (29.7\%). These findings were similar to the study of Mansour et al (2020) and Chen et al (2017) in which most of the participants (25.4\%) willing to perform CPR only on family members, (76.3\%) on strangers, however, $53.2 \%$ respondents were anxious due to legal issues and $44.4 \%$ were worried due to inadequate knowledge. In addition to this, response regarding legal issues in performing CPR into account depicted the fact that most students (31\%) believed to prosecute CPR as liability if bystander CPR have to perform on their family members failed whereas $38.6 \%$ were uncertain about it.

Consecutively, more than half of the students $(68.6 \%)$ agreed to pay for the qualified and professional training, followed by not agreed (11.9\%) and (19.9\%) were not sure about the answer. Furthermore, $84.7 \%$ think that CPR training should be mandatory for all the students in undergraduate program, nonetheless these ratios are comparatively lower in comparison to the findings of Aroor et al1 and Ghauri et al26. Besides, majority of students in our study think best method-in their opinionto increase public awareness of the importance of CPR is to inform them about the availability of free training courses (61.9\%) and campaigns (23.7\%). Despite of the findings, all participants showed willingness to learn and perform CPR therefore substantial curriculum should be incorporated for better healthcare delivery.

To the best of author's knowledge, this is the first study to explore the level of knowledge and attitude among rehab sciences students towards bystander CPR in Pakistan. The study provides great insight towards the CPR domain and its implementation in the field.

Number of limitations has been encountered in this study included the limited sample size due to ongoing COVID pandemic. Moreover, majority of students did not fill some question due to unknown reasons that may cause uncertainty in the results.

The findings suggested that CPR training course should be made mandatory for undergraduate medical students and the training program is advised to be incorporated in the curriculum so that the effective application of technique will be made possible for better healthcare 
delivery. Future studies should involve more survey that also analyses the practice of CPR to rule out the education gap in undergraduate medical students.

\section{CONCLUSION}

It was concluded that the majority of students have inadequate knowledge regarding $C P R$, however positive attitude and willingness to learn the correct protocol was observed. Therefore, a certified and updated training program is recommended that should be incorporated as an essential component in an undergraduate medical curriculum.

\section{REFERENCES}

[1] Aroor AR, Saya RP, Attar NR, Saya GK, Ravinanthanan M. Awareness about basic life support and emergency medical services and its associated factors among students in a tertiary care hospital in South India. Journal of emergencies, trauma, and shock. 2014 Jul;7(3):166.

[2] World Health Organization, Eastern Mediterranean status report on road safety: call for action. 2010.

[3] Irfan B, Zahid I, Khan MS, Khan OA, Zaidi S, Awan S, Bilal S, Irfan O. Current state of knowledge of basic life support in health professionals of the largest city in Pakistan: a crosssectional study. BMC health services research. 2019 Dec;19(1):1-7.

[4] Ebell $\mathrm{MH}$, Afonso AM. Pre-arrest predictors of failure to survive after in-hospital cardiopulmonary resuscitation: a meta-analysis. Family practice. 2011 Oct 1;28(5):505-15.

[5] Ativie RN, Awotidebe TO, Omeru O, Ogunyemi SA, Olowe GT, Adedoyin RA, Folarin ET. Knowledge, Attitude and Experience of Cardiopulmonary Resuscitation among Medical and Healthcare Professional Students in a Nigerian Medical College. Journal of Advances in Medicine and Medical Research. 2018 Dec 8:1-9.Onyeaso
[6] A.O. and Onyeaso, O.O. Knowledge of Cardiopulmonary Resuscitation among Student Teachers in Nigeria. Journal of Health Science, [7(5),2017).pp.91-95.

http://article.sapub.org/10.5923.j.hea Ith.20170705.01.html.

[7] Abella BS, Sandbo N, Vassilatos P, Alvarado JP, O'Hearn N, Wigder HN, Hoffman P, Tynus K, Vanden Hoek TL, Becker LB. Chest compression rates during cardiopulmonary resuscitation are suboptimal: a prospective study during in-hospital cardiac arrest. circulation. 2005 Feb 1;111(4):428-34.

[8] Solagberu BA. Knowledge and practice of cardiopulmonary resuscitation among Nigerian doctors. Nigerian Journal of Surgical Research. 2002;4(1):12-21.

[9] Rajapakse R, Noč M, Kersnik J. Public knowledge of cardiopulmonary resuscitation in Republic of Slovenia. Wiener Klinische Wochenschrift. 2010 Dec;122(23):667-72.

[10] Holmberg S, Handley A, Bahr J, Baskeit $P$, Bossaert L, Chamberlain D, Dick W, van Drenth $A$, Ekstrom $L$, Juchems $R$, Kettler D. Guidelines for basic life support: a statement by the Basic Life Support Working Party of the European Resuscitation Council, 1992. Resuscitation. 1992 Nov 1;24(2):10310.

[11] Chen, M., Wang, Y., Li, X., Hou, L., Wang, Y., Liu, J. and Han, F., 2017. Public Knowledge and Attitudes towards Bystander Cardiopulmonary Resuscitation in China. BioMed Research International, 2017, pp.1-7.

[12] Kanstad BK, Nilsen SA, Fredriksen KC. CPR knowledge and attitude to performing bystander CPR among secondary school students in Norway. Resuscitation. 2011 Aug 1;82(8):10539.

[13] N. Mpotos, E. Vekeman, K. Monsieurs, A. Derese, and M. Valcke, "Knowledge and willingness to teach cardiopulmonary resuscitation: a 
survey amongst 4273 teachers," Resuscitation, vol. 84, no. 4, pp. 496500, 2013.

[14] Bogle B, Mehrotra S, Chiampas G, Aldeen AZ. Assessment of knowledge and attitudes regarding automated external defibrillators and cardiopulmonary resuscitation among American University students. Emergency Medicine Journal. 2013 Oct 1;30(10):837-41.

[15] Hamasu S, Morimoto T, Kuramoto N, Horiguchi M, Iwami T, Nishiyama C, Takada K, Kubota Y, Seki S, Maeda Y, Sakai $Y$. Effects of BLS training on factors associated with attitude toward CPR in college students. Resuscitation. 2009 Mar 1;80(3):35964.

[16] Lu C, Jin Y, Meng F, Wang Y, Shi X, Ma W, Chen J, Zhang $Y$, Wang W, Xing $Q$. An exploration of attitudes toward bystander cardiopulmonary resuscitation in university students in Tianjin, China: a survey. International emergency nursing. 2016 Jan 1;24:2834.

[17] Alshaer H, Fernie GR, Bradley TD. Phase tracking of the breathing cycle in sleeping subjects by frequency analysis of acoustic data. International Journal of Healthcare Technology and Management. 2010 Jan 1;11(3):16375.

[18] Chandrasekaran S, Kumar S, Bhat SA. Awareness of basic life support among medical, dental, nursing students and doctors. Indian journal of Anaesthesia. 2010 Mar;54(2):121.

[19] Ma A, Wong KL, Tou AY, Vyas L, Wilks J. CPR knowledge and attitudes among high school students aged 15-16 in Hong Kong. Hong Kong Journal of Emergency Medicine. 2015 Jan;22(1):3-13.

[20] Owaid Alsharari A, Alduraywish A, Ali Al-Zarea E, Ibrahim Salmon N, Ali Sheikh MS. Current status of knowledge about cardiopulmonary resuscitation among the university students in the northern region of Saudi Arabia. Cardiology research and practice. 2018 Jun 10;2018.

[21] Özbilgin Ş, Akan M, Hancı V, Aygün C, Kuvaki B. Evaluation of public awareness, knowledge and attitudes about cardiopulmonary resuscitation: report of İzmir. Turkish journal of anaesthesiology and reanimation. 2015 Dec;43(6):396.

[22] Majid A, Jamali $M$, Ashrafi MM, Haq ZU, Irfan R, Rehan A, Memon MM, Khan MA, Kumar J, Singh PK, Luis SA. Knowledge and attitude towards cardiopulmonary resuscitation among doctors of a tertiary care hospital in Karachi. Cureus. 2019 Mar;11(3).

[23] Mansour A, Alsager AH, Alasqah A, Altamimi AS, Alsuhaibani A, Aljabr AA, AlDughaim Al. Student's Knowledge, Attitudes, and Practices Related to Cardiopulmonary Resuscitation at Qassim University, Saudi Arabia. Cureus. 2019 Nov;11(11).

[24] Pepera G, Xanthos E, Lilios A, Xanthos $T$. Knowledge of cardiopulmonary resuscitation among Greek physiotherapists. Monaldi Archives for Chest Disease. 2019 Nov 12;89(3).

[25] Ghauri SK, Javaeed A, Shah F. Dismal situation of cardio pulmonary resuscitation knowledge and skills among junior doctors in twin cities of Pakistan. Pakistan Journal of Medical Sciences. 2019 Sep;35(5):1295. 